\title{
Antiretroviral drug resistance among U.S. veterans living with human immunodeficiency virus 1
}

\author{
Rahul Mahapatra ${ }^{1}$, Nancy Barrett ${ }^{2}$, George Psevdos ${ }^{2}$ \\ ${ }^{1}$ Stony Brook University Hospital, Stony Brook, United States \\ ${ }^{2}$ Veterans Affairs Medical Center, Northport, United States
}

\begin{abstract}
Introduction: Resistance to antiretroviral medications poses challenges for the successful treatment of human immunodeficiency virus 1 (HIV-1) infection. Genotypic antiretroviral testing provides guidance for selecting the proper treatment regimens. We studied the prevalence of HIV-1 resistance mutations in a population of U.S. veterans.

Material and methods: A retrospective chart review was performed on 230 patients who presented to an outpatient infectious diseases clinic for routine HIV-1 care between 2000 and 2016. Charts were reviewed to extract available information on genotype test results and relevant demographic data.

Results: Of the 230 patients, 98 had available genotype tests. A total of 113 genotype tests were collected for analysis. Fifty-three genotypes were baseline tests; 60 were obtained following virologic failure. The median age of the study group was 58 years. Ninety-four of the 98 subjects were men. Risk factors for HIV-1 acquisition included intravenous drug use (31\%) and unprotected heterosexual (27\%) and homosexual (24\%) encounters. At the end of the follow-up period, CD4+ T-cell median was 557/ $\mu \mathrm{l}$ and HIV-1 viral load median was 20 copies/ml. K103N was seen in 2 baseline tests. The most common acquired resistance mutations were M184V (70\%), K103N (55\%), and thymidine analogue mutations (TAM). There was 1 patient with integrase strand transfer inhibitor (INSTI) mutation. Virologic control among patients with acquired resistance was achieved with protease inhibitor (PI) based or PI-(INSTI)-combined regimens.
\end{abstract}

Conclusions: M184V, K103N, and TAM were the most common resistance mutations. INSTI mutation was seen in only 1 patient. PI and PI-INSTI combinations achieved HIV-1 viral load suppression in patients with resistance mutations.

HIV AIDS Rev 2019; 18, 1: 57-61

DOI: https://doi.org/10.5114/hivar.2019.84202

Key words: HIV infection, antiretroviral drugs, drug resistance, veterans.

\section{Introduction}

Over 27,000 United States (U.S.) veterans are living with human immunodeficiency virus 1 (HIV-1) infection [1]. In 1981, HIV-1 infection was recognized and the ensuing epi-

Address for correspondence: Dr. George Psevdos, Veterans Affairs Medical Center, 79 Middleville Road, 11768, Northport,

United States, e-mail: george.psevdos@va.gov

demic of acquired immune deficiency syndrome (AIDS) was synonymous with a death sentence. The advent of antiretroviral therapy was followed immediately by viral genotypic resistance, necessitating the need for new classes of antiretroviral medications and increasingly complex regimens [2].

Article history:

Received: 23.02.2018

Received in revised form: 08.05.2018

Accepted: 14.09.2018

Available online: 15.03.2019
International Journal of HIV-Related Problems

HIV \& AIDS R e v i e w 
In 1996, with the development of highly active antiretroviral therapy (HAART), treatment of the HIV-1 infection with sustained virologic suppression became possible, forever altering the course of the disease. It is currently accepted practice to perform baseline antiretroviral resistance testing in newly diagnosed patient with HIV-1 infection as well as at time of treatment failure.

There are two methods for estimating the susceptibility of HIV-1 samples replicated in the patient's plasma: genotypic and phenotypic. Phenotypic assays can detect the susceptibility of HIV-1 to an antiretroviral drug while genotypic assays are used to detect mutations associated with established HIV-1 resistance. These methods can be performed at baseline before initiation of therapy or at virologic failure (i.e., the inability to achieve or maintain suppression of viral replication while on HAART). Genotypic assays are based on sequencing of nucleotides of the HIV-1 genes (the pol gene, for example, which encodes HIV-1 reverse transcriptase amino acid sequences) with identification of patterns of HIV-1 mutations associated with antiretroviral resistance [3]. Resistance mechanisms can be complex, involving interactions between mutations and their associated pathways. Their impact on cross-resistance within antiretroviral classes greatly influences treatment decisions [4].

There are few reports assessing HIV-1 antiretroviral drug resistance among U.S. veterans. Soto-Pillich et al. analyzed resistance mutations in veterans living in Puerto Rico under the San Juan Veterans Affairs (VA) healthcare system; they found M184V and $\mathrm{K} 103 \mathrm{~N}$ as the most frequent mutations [5]. A six-year retrospective, cross-sectional study in the District of Columbia, between 2001 and 2006, revealed a reduction in the frequency of nucleoside reverse transcription inhibitor (NRTI) and non-nucleoside reverse transcription inhibitor (NNRTI) resistance mutations among veterans living with HIV-1 infection on HAART [6]. In the same study, there was a rise in resistance mutations of the protease inhibitor (PI) atazanavir. These findings mirrored antiretroviral (ART) drug utilization among patients in the study with pivotal impacts on ART treatment and transmission of viral resistance in a high-prevalence community [6]. In our retrospective cross-sectional analysis, we identified genotypic mutations in U.S. veterans living with HIV/AIDS in both treatment-naïve and treatment-experienced patients.

\section{Material and methods}

A retrospective chart review was conducted from January $1^{\text {st }}, 2000$ to December $31^{\text {st }}, 2016$. In this cross-sectional analysis demographic data were collected, such as age, sex, race, as well as risk factors for acquisition of HIV-1, genotypes at baseline and at treatment failures, comorbidities, AIDS-defining conditions, CD4 ${ }^{+}$T-helper cell counts with HIV-1 viral loads at the end of follow-up. We determined the prevalence of drug-resistant mutations and analyzed how many patients remained viremic at the end of follow-up. Frequencies were calculated using Microsoft Excel. The study was approved by the Institutional Review Board (approval number 00474) of the Northport VA Medical Center (VAMC).

All patients were U.S. veterans 18 years old or older. All had been diagnosed with HIV-1 infection via confirmatory testing, either western blot or indirect immunofluorescence assay. The patient's HIV-1 RNA was extracted from plasma samples and amplified in a reference laboratory within the Veterans Affairs system. Until 2015 genomic HIV-1 mutations were identified using the TRUGENE HIV-1 genotyping system, Siemens, St. Denis, France. Since 2015, the ViroSeq HIV-1 Genotyping System, Abbot Laboratories, Illinois, USA, has been utilized. These systems are designed to perform population sequencing of the reverse transcriptase, protease, and integrase segment of the pol gene of HIV-1. Interpretation of mutations was based on the International Antiviral Society-USA (IAS-USA) Drug Resistance Mutation Group's list of evidence-based information on drug-resistance associated mutations [7]. NRTI resistance mutations were divided into non-thymidine analogue mutations, M184V/I, K65R, K70R/E, L74V/I, Y115F; multiple drug resistant Q151M, T69ins, and thymidine analogue mutations (TAM), M41L, D67N/G/E, L210W, T215Y/F, and K219Q/E. NNRTI associated mutations included L100I, K101E/P, K103N/S, V106A/M, E138K, V179F, Y181C/I/V, Y188L/ $\mathrm{C} / \mathrm{H}, \mathrm{G} 190 \mathrm{~S} / \mathrm{A} / \mathrm{E}$, and P225H. PI resistance mutations included L10I, K20R, L24I, V32I, L33F, D30N, E35D, M36I, M46I/L, I47V/A, G48V/M, I50L, I54M, I62V, L63P, A71T/ V/L, G73S/T/C, V77I, V82A/S/T, I84V, and L90M. INSTI associated mutations included T66I/K, E92Q, Y143R/H/C, S147G, Q148H/K/R, and N155N. All patients were followed in the Infectious Diseases Clinic at Northport VAMC. Veterans who left our institution and established continuity care at other VA facilities were excluded from the analysis.

\section{Results}

Two hundred thirty U.S. veterans living with HIV-1 were identified during the study period of 2000-2016 (see Figure 1). Thirty patients established continuity care at other VA facilities and were excluded. Three were "elite controllers" and their HIV-1 viral load remained undetectable without breakthrough viremia. Seventy-seven had controlled HIV-1 infection throughout the study period and had no baseline genotypes. Ninety-eight patients had genotype data available, with 113 total genotype assays. Twenty-two had evidence of viremia at the end of follow-up but had no genotype data either due to death or lack of follow-up. Table 1 shows the demographic characteristics of the 98 patients with genotype data. Of the 113 genotypes, 53 were baseline, 50 on $1^{\text {st }}$ antiretroviral failure, 9 on $2^{\text {nd }}$ failure and 1 on $3^{\text {rd }}$ failure. The median nadir CD4 ${ }^{+}$T-cell count was $191 / \mu$ l. Thirty out of 98 patients, or $31 \%$, had a history of an AIDS defining illness. Table 2 depicts frequencies of the baseline and acquired resistance mutations. Figures 2-4 show NRTI, NNRTI and PI mutations. From the 53 baseline genotypes L63P was seen in 23, M36I in 13, V771 in 9; K103N mutation was seen in 2 patients. No M184V mutation detected on baseline testing. 


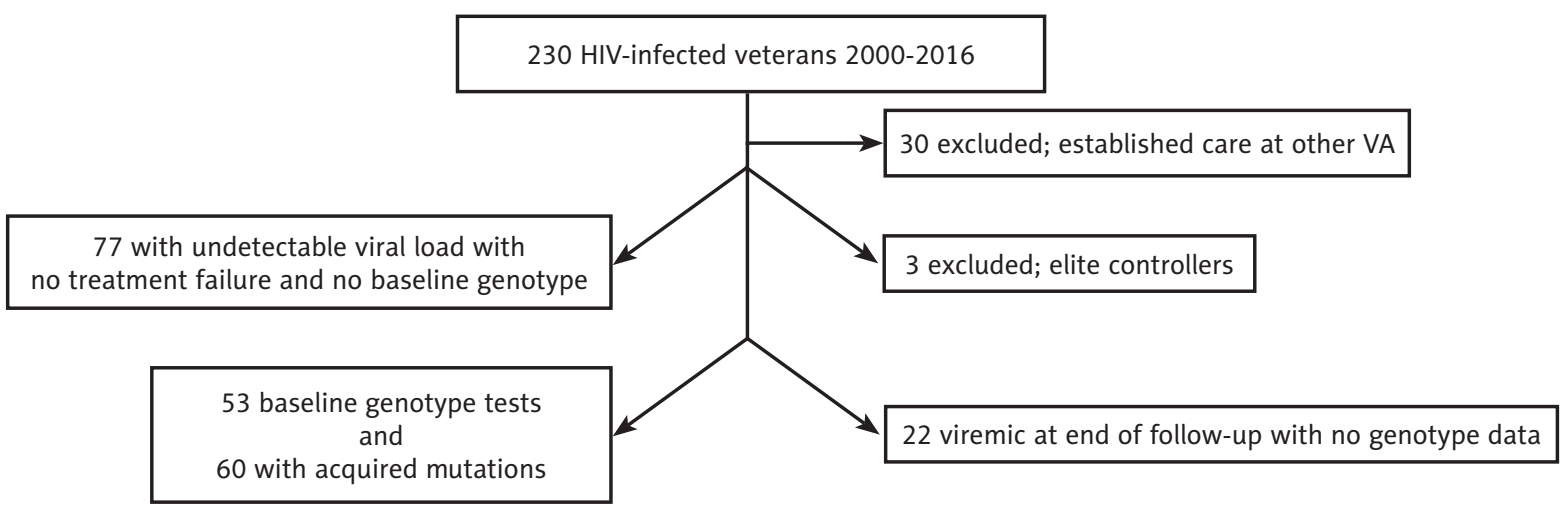

Figure 1. Study flowchart

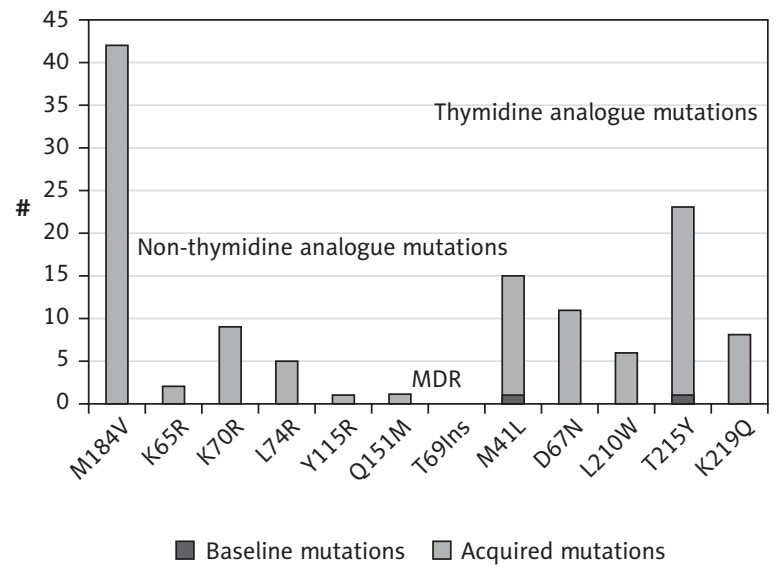

Figure 2. Nucleoside reverse transcription inhibitor - resistant mutations (MDR multidrug resistant)

The most common acquired mutations were: $\mathrm{M} 184 \mathrm{~V}$ in 42 (70\%), L63P in $36(60 \%), \mathrm{K} 103 \mathrm{~N}$ in 33 (55\%), and $\mathrm{T} 215 \mathrm{Y}$ in $22(36 \%)$. K65R mutation was noted only in 2 patients, Q151M in 1, and I50L in 1 patient. There was only 1 case of integrase strand inhibitor resistance (patient on elvitegravir-containing regimen), E92Q. At the end of follow-up, the median HIV-1 viral load was 20 copies/ml. Only 15 patients had serum HIV-1 viral loads greater than 500 copies/ $\mathrm{ml}$ (range 509-728,000). Patients who achieved virologic control after treatment failures used the following antiretroviral regimens: protease inhibitor-based regimen $40 \%$, integrase strand inhibitors (INSTI) + PI based 32\%, INSTI + PI + NNRTI $11 \%$, NNRTI $8 \%$, NNRTI + PI 3\%, INSTI 3\%, and NRTI only $3 \%$.

\section{Discussion}

In its 2012 global HIV-1 drug resistance report, the World Health Organization estimated that $10-17 \%$ of antiretroviral naïve HIV-1 infected individuals in Australia, Japan, Europe and the United States had acquired a virus that was resistant to at least one antiretroviral drug [8]. In our cohort, important baseline resistance mutations were seen in 2 cases

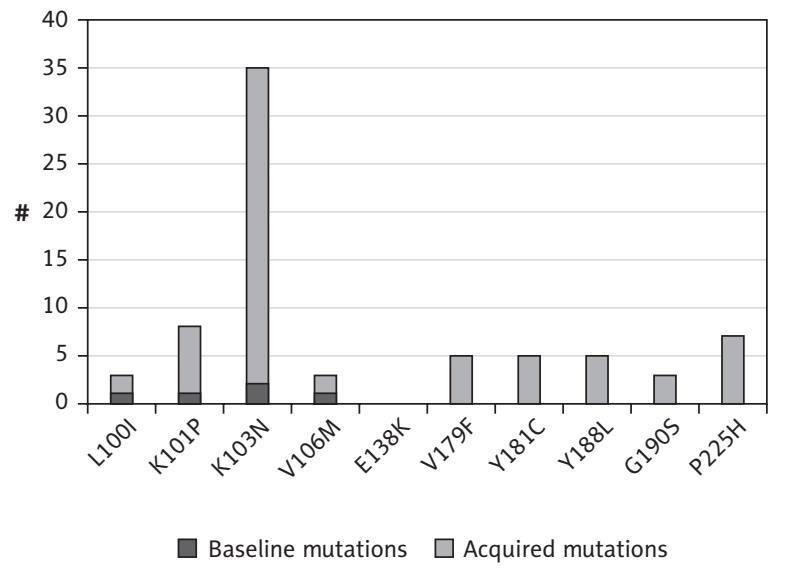

Figure 3. Non-nucleoside reverse transcription inhibitor - resistant mutations

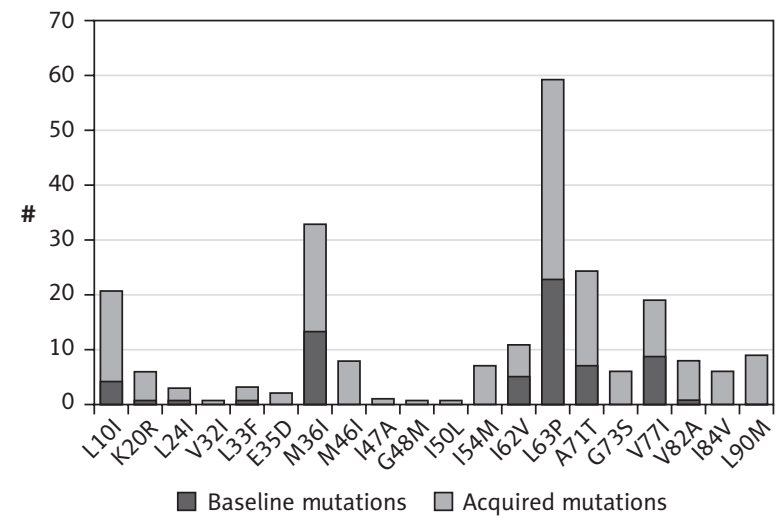

Figure 4. Protease inhibitor - resistant mutations

with presence of K103N. Margot et al. reported from 2000 to 2013 a $4.5 \%$ pretreatment resistance rate with NNRTI, notably K103N/S, in ART-naïve patients [9]. In our study, baseline genotype data were unavailable in a significant number of patients. Many of them were initiated on treatment without baseline testing as it was not readily available, especially in the early years of the study period. Others entered the study 
Table 1. Demographic characteristics of 98 patients with genotype data

\begin{tabular}{|c|}
\hline Factor \\
\hline Median age: 58 years (range: $28-82$, IQR 13 ) \\
\hline Median years of HIV-1 infection: 14 (range 1-31, IQR 2) \\
\hline Men $(\#): 94$ \\
\hline Women $(\#): 4$ \\
\hline African American: 56 (57\%) \\
\hline Caucasian: 41 (42\%) \\
\hline American Indian: 1 (1\%) \\
\hline HIV risk factors: \\
\hline IVDU: 30 (31\%) \\
\hline Heterosexual: 27 (27\%) \\
\hline MSM: 24 (25\%) \\
\hline Blood transfusion: 3 (3\%) \\
\hline Unknown: 14 (14\%) \\
\hline $\begin{array}{l}\text { Median nadir CD4+ T-cells (cells/ul): } 191 \text { (range 1-728, } \\
\text { IQR 274.5) }\end{array}$ \\
\hline 30 (31\%) had AIDS-related diagnoses \\
\hline Pneumocystis pneumonia: 10 (cases) \\
\hline Oral thrush with presumptive esophageal involvement: 12 \\
\hline Candida esophagitis (biopsy proven): 2 \\
\hline Other: $6^{*}$ \\
\hline $\begin{array}{l}\text { Median CD4+ T-cells at end of follow-up (cells/ } \mu \text { l): } 557 \\
\text { (range 4-2033, IQR 509.25) }\end{array}$ \\
\hline $\begin{array}{l}\text { Median HIV viral load at end of follow-up (copies/ml): } 20 \\
\text { (range }<20-728,000 \text {, IQR 56.25) }\end{array}$ \\
\hline HCV co-infection: 37 (38\%) \\
\hline HCV genotypes** \\
\hline HBV co-infection: 3 (3\%) \\
\hline 113 available genotype tests \\
\hline 53 baseline \\
\hline 60 upon virologic failure \\
\hline $\begin{array}{l}\text { AIDS - acquired immunodeficiency syndrome, HIV - human } \\
\text { immunodeficiency virus, HCV - hepatitis C virus, HBV - hepatitis B virus, } \\
\text { IQR - interquartile range, IVDU - intravenous drug use, MSM - men who } \\
\text { have sex with men } \\
\text { *Other: one case each of cerebral toxoplasmosis, cytomegalovirus retinitis, } \\
\text { tuberculosis, Kaposi sarcoma, non-Hodgkin's lymphoma and eosinophilic } \\
\text { folliculitis. } \\
{ }^{* *} H C V \text { genotypes seen: } 1 a-14(38 \%), 1 b-11(30) \%, 2 a-1(3 \%), \\
3 a-1(3 \%) \text { genotype undetermined } 10(26 \%) \text {. }\end{array}$ \\
\hline
\end{tabular}

cohort already on treatment and virally suppressed. The high prevalence of acquired M184V and K103N mutations in our study group was expected and consistent with prior reports. Not unexpectedly, the majority of our patients (93 out of 98) were treated with lamivudine (3TC) and emtricitabine (FTC). 3TC and FTC are NRTIs commonly used as part of the "backbone" of ART (two NRTIs plus an NNRTI, or PI or INSTI). Failing ART that contains 3TC or FTC, the M184V mutation
Table 2. Overall encountered mutations and their frequencies

\begin{tabular}{l|c|c}
\hline \multirow{2}{*}{ Mutations } & Baseline $(n, \%)$ & Acquired $(n, \%)$ \\
\cline { 2 - 3 } & $n=53$ genotype tests & $n=60$ genotype tests \\
\hline RT mutations & \multicolumn{2}{|c}{} \\
\hline M41L & $1(2 \%)$ & $14(23 \%)$ \\
\hline D67N & 0 & $11(18 \%)$ \\
\hline K70R & 0 & $9(15 \%)$ \\
\hline L74V & 0 & $5(8 \%)$ \\
\hline K65R & 0 & $2(3 \%)$ \\
\hline M184V & 0 & $42(70 \%)$ \\
\hline Y115F & 0 & $1(2 \%)$ \\
\hline T215Y & $1(2 \%)$ & $22(36 \%)$ \\
\hline L210W & 0 & $6(10 \%)$ \\
\hline Q151M & 0 & $1(2 \%)$ \\
\hline K101P & $1(2 \%)$ & $7(11 \%)$ \\
\hline K103N & $2(4 \%)$ & $33(55 \%)$ \\
\hline Y181C & 0 & $5(8 \%)$ \\
\hline Y188C & 0 & $5(8 \%)$ \\
\hline G190S & 0 & $3(5 \%)$ \\
\hline P225H & 0 & $7(11 \%)$ \\
\hline PItan & & \\
\hline M & 0 & \\
\hline
\end{tabular}

PI mutations

\begin{tabular}{l|c|c}
\hline L24I & $1(2 \%)$ & $2(3 \%)$ \\
\hline V32I & 0 & $1(2 \%)$ \\
\hline L33F & $1(2 \%)$ & $2(3 \%)$ \\
\hline D30N & 0 & 0 \\
\hline M46I & $1(2 \%)$ & $8(13 \%)$ \\
\hline I47V & 0 & $1(2 \%)$ \\
\hline I50L & 0 & $1(2 \%)$ \\
\hline I54V & 0 & $7(11 \%)$ \\
\hline L63P & $23(43 \%)$ & $36(60 \%)$ \\
\hline A71T & $7(13 \%)$ & $17(23 \%)$ \\
\hline G73S & 0 & $6(10 \%)$ \\
\hline V77I & $9(17 \%)$ & $10(16 \%)$ \\
\hline I84V & 0 & $6(10 \%)$ \\
\hline L89V & $1(2 \%)$ & $3(5 \%)$ \\
\hline L90M & 0 & $9(15 \%)$ \\
\hline INSTI mutations & 0 & $1(2 \%)$ \\
\hline E92Q & 0 & \\
\hline
\end{tabular}

$R T$ - reverse transcriptase, $\mathrm{PI}$ - protease inhibitor, INSTI - integrase strand transfer inhibitor

is usually the first to appear [10]. K103N is the most common NNRTI mutation and may occur as the first resistance mutation, even preceding M184V [10].

Resistance mutations to zidovudine (AZT) or stavudine (D4T) are known as thymidine analogue mutations (TAM). Acquired TAM were prevalent in our cohort as many of our 
patients were older and had been living with HIV-1 for many years, were treatment experienced and were likely exposed to AZT or D4T in their initial treatments. The incidence of TAM is expected to decrease as $\mathrm{AZT}$ and $\mathrm{D} 4 \mathrm{~T}$ are no longer recommended as initial choices by the Department of Health and Human Services (DHHS) in their guidelines for the use of antiretroviral agents in adults and adolescents living with HIV-1 [11]. Tenofovir (K65R), protease inhibitor and integrase strand transfer inhibitors mutations were infrequent in our cohort. No signature mutation (E138K) was detected for the NNRTI rilpivirine. Indeed most of our patients who developed resistance mutations were eventually controlled, often with complex constructed antiretroviral regimens with protease inhibitor or INSTI-protease inhibitor combinations.

The use of INSTI-based regimens for initial therapy in antiretroviral naïve persons living with HIV-1 is increasing as they are the recommended initial therapy in the DHHS guidelines, and are gradually replacing PI and NNRTIs within first-line regimens [11]. Frange et al. reported a low frequency of INSTI resistance in a French cohort, and the You et al. meta-analysis showed low rates of resistance to raltegravir (3.9\%) and elvitegravir $(1.2 \%)[12,13]$. Some experts suggest INSTI screening in ART-naïve patients before initiation to exclude possible transmission of resistance [14]. It is important for the clinician to understand which genotypic test is routinely utilized and whether or not INSTI sequences are included. Referral to an experienced reference laboratory may be considered. Indeed, appropriate selection and sequencing of therapies is the most efficacious and cost-effective method of managing lifelong therapy for HIV-1 at a population level [14].

Nearly $40 \%$ of our patients were co-infected with hepatitis $\mathrm{C}(\mathrm{HCV})$. This is a higher reported rate compared to the approximately 25\% HIV-HCV co-infection rate seen nationally in the United States [15]. In our cohort, we documented a $38 \%$ co-infection rate, which is similar to the $40 \%$ reported by Backus et al. among HIV-HCV co-infected U.S. veterans [16]. This is not surprising, as intravenous drug use was reported in one third of our veteran population.

\section{Conclusions}

Antiretroviral resistance testing to guide therapy selection in ART-naïve and treatment-experienced patients with virologic failure is widely accepted as a standard of care in managing HIV-1 infection. The most prevalent HIV-1 resistance mutations in our U.S. veteran population were M184V and $\mathrm{K} 103 \mathrm{~N}$ and TAM. Tenofovir and integrase inhibitor resistance mutations were rare. As new antiretrovirals and single tablet complete regimens are being developed, epidemiologic studies should continue among U.S. veterans living with HIV-1 to detect trends in resistance to individual classes which may impact subsequent therapy options.

\section{Acknowledgements}

The authors would like to thank Dr. Asrat Tesfa for reviewing the manuscript.

\section{Conflict of interest}

The authors declare no potential conflicts of interest with respect to the research, authorship, and/or publication of this article.

\section{References}

1. Veterans Health Administration focuses on Veterans and HIV prevention. Available at: https://www.blogs.va.gov/VAntage/ 33227/world-aids-day-2016-vha-focuses-veterans-hiv-prevention/ (Accessed: 02.01.2018).

2. Wainberg MA, Rooke R, Tremblay M, et al. Clinical significance and characterization of AZT-resistant strains of HIV-1. Can J Infect Dis 1991; 2: 5-11.

3. Durant J, Clevenbergh P, Halfon P, et al. Drug-resistance genotyping in HIV-1 therapy: the VIRADAPT randomized controlled trial. Lancet 1999; 353: 2195-2199.

4. Tang MW, Shafer RW. HIV-1 antiretroviral resistance: scientific principles and clinical application. Drugs 2012; 72: e1-e25.

5. Soto-Pillich GE, Santiago-Vazquez A, Calderon-Vizcarrondo, et al. Prevalence of antiretroviral drug-resistance mutations in HIV-1 positive treatment naïve and treatment-experienced Puerto Rican Veterans from San Juan VA Caribbean Healthcare System. Infect Dis Clin Pract 2015; 23: 324-326.

6. Arastu AA, Kan V. Characterization of genotypic mutations and antiretroviral resistance among viremic HIV-1 infected patients in a high HIV prevalence area: treatment challenge and transmission risk. World J AIDS 2011; 1: 70-77.

7. Wensing AM, Calvez V, Günthard HF, et al. 2017 Update of the Drug Resistance Mutations in HIV-1. Top Antivir Med 2017; 24: 132-133.

8. WHO HIV Drug Resistance Report 2012. Available at: http://apps. who.int/iris/bitstream/10665/75183/1/9789241503938_eng.pdf (Accessed: 02.01.2018).

9. Margot NA, Wong P, Kulkarni R, et al. Commonly transmitted HIV-1 drug resistance mutations reverse-transcriptase and protease in antiretroviral treatment-naïve patients and response to regimens containing tenofovir disoproxil fumarate or tenofovir alafenamide. J infect Dis 2017; 215: 920-927.

10. Gallant JE. Antiretroviral drug resistance and resistance testing. Top HIV Med 2005; 12: 138-142.

11. Panel on Antiretroviral Guidelines for Adults and Adolescents. Guidelines for the Use of Antiretroviral Agents in Adults and Adolescents living with HIV. Department of Health and Human Services. Available at: http://www.aidsinfo.nih.gov/ContentFiles/ AdultandAdolescentGL.pdf (Accessed: 02.05.2018).

12. Frange P, Assoumou L, Descamps D, et al. HIV-1 subtype B-infected MSM may have driven the spread of transmitted resistant strains in France in 2007-12: impact on susceptibility to first-line strategies. J Antimicrob Chemother 2015; 70: 2084-2089.

13. You J, Wang H, Huang X, et al. Therapy-emergent drug resistance to Integrase strand transfer inhibitors in HIV-1 patients: a subgroup meta-analysis of clinical trials. PLoS One 2016; 17: 11.

14. Boucher CA, Bobkova MR, Geretti AM, et al. State of the antiretroviral therapy in human immunodeficiency virus drug resistance: science and technology knowledge gap. AIDS Rev 2018: 20: 26-41.

15. Kim JH, Psevdos G, Suh J, Sharp VL. Co-infection of hepatitis B and hepatitis $\mathrm{C}$ virus in human immunodeficiency virus-infected patients in New York City, United States. World J Gastroenterol 2008; 14: 6689-6693.

16. Backus L, Phillips B, Boothroyd D, et al. Hepatitis C coinfection increases mortality in HIV-infected U.S. veterans treated with highly active antiretroviral therapy. Abstract. $11^{\text {th }}$ Conference on Retroviruses and Opportunistic Infections. San Francisco, 2004. 